\title{
$k$-REGULAR EMBEDDINGS OF THE PLANE
}

\author{
F. R. COHEN $^{1}$ AND D. HANDEL
}

\begin{abstract}
A map $f: X \rightarrow R^{n}$ is said to be $k$-regular if whenever $x_{1}, \ldots, x_{k}$ are distinct points of $X$, then $f\left(x_{1}\right), \ldots, f\left(x_{k}\right)$ are linearly independent. Such maps are of interest in the theory of Cebysev approximation. In this paper, configuration spaces and homological methods are used to show that there does not exist a $k$-regular map of $R^{2}$ into $R^{2 k-\alpha(k)-1}$ where $\alpha(k)$ denotes the number of ones in the dyadic expansion of $k$. This result is best possible when $k$ is a power of 2 .
\end{abstract}

1. Introduction. Let $k \leqslant n$ be positive integers. A continuous map $f$ : $X \rightarrow R^{n}$ is $k$-regular if whenever $x_{1}, \ldots, x_{k}$ are distinct points of $X$, then $f\left(x_{1}\right), \ldots, f\left(x_{k}\right)$ are linearly independent.

EXAMPLE 1.1. $f: R \rightarrow R^{k}$ given by $f(t)=\left(1, t, t^{2}, \ldots, t^{k-1}\right)$ is $k$-regular, as is seen by the nonvanishing of the Vandermonde determinant.

EXAMPLE 1.2. $f: R^{2}=C \rightarrow R^{2 k-1}=R \times C^{k-1}$ given by $f(z)=$ $\left(1, z, \mathrm{z}^{2}, \ldots, z^{k-1}\right)$ is $k$-regular.

$k$-regular maps are of relevance in the theory of Čebyšev approximation. The connection is as follows: Let $X$ be a compact subspace of some Euclidean space, and suppose $f_{1}, \ldots, f_{n}$ are continuous, linearly independent real-valued functions defined on $X$. For an arbitrary continuous $g: X \rightarrow R$, let $\mathscr{B}(g)$ denote the set of best approximations to $g$ by linear combinations of $f_{1}, \ldots, f_{n}$, i.e. if $F$ denotes the $n$-dimensional vector space of functions spanned by the $f_{i}$ and $m_{g}=\inf _{f \in F} \sup _{x \in X}|g(x)-f(x)|$, then $\mathscr{B}(g)=\{f \in$ $\left.F: \sup _{x \in X}|g(x)-f(x)|=m_{g}\right\}$. Then the following theorem holds:

TheOREM (HAAR-Kolmogorov-RubinsteIn). For every continuous $g: X \rightarrow$ $R$, the dimension of the set $\mathscr{B}(g)$ is $\leqslant n-k$ if and only if $\left(f_{1}, \ldots, f_{n}\right)$ : $X \rightarrow R^{n}$ is $k$-regular.

For a proof see [9, pp. 237-242].

For example, $f=\left(f_{1}, \ldots, f_{n}\right): X \rightarrow R^{n}$ is $n$-regular if and only if $f_{1}, \ldots, f_{n}$ form a Haar system on $X$, i.e. $\operatorname{det}\left(f_{i}\left(x_{j}\right)\right) \neq 0$ whenever $x_{1}, \ldots, x_{n}$ are distinct points of $X$. This is the case if and only if every continuous $g: X \rightarrow R$ has a unique best approximation by linear combinations of the $f_{i}$.

Work on existence and nonexistence of $k$-regular maps by nonalgebraic

Presented to the Society, August 8, 1977; received by the editors June 21, 1977.

AMS (MOS) subject classifications (1970). Primary 55G99; Secondary 41A50.

$K e y$ words and phrases. $k$-regular maps, configuration spaces, Dyer-Lashof operations, StiefelWhitney classes.

'Partially supported by a National Science Foundation grant. 
topological methods appears in [1], [2], and [6]. For example, in [1], the following is proved:

THEOREM 1.3 (BOLTJANSKIĪ-RYŠKOV-ŠAŠKIN). If a $2 k$-regular map of $R^{n}$ into $R^{N}$ exists, then $N \geqslant(n+1) k$.

In fact, if $f: R^{n} \rightarrow R^{N}$ is $2 k$-regular, then if $E_{1}, \ldots, E_{k}$ are pairwisedisjoint $n$-discs in $R^{n}$, the map $g: E_{1} \times \cdots \times E_{k} \times(R-\{0\})^{k} \rightarrow R^{N}$ given by $g\left(x_{1}, \ldots, x_{k} ; t_{1}, \ldots, t_{k}\right)=\sum_{i} t_{i} f\left(x_{i}\right)$ is injective, and so $N$ cannot be less than the dimension of the space on the left.

In [5], homological methods, using configuration spaces, are used to get a nonexistence result. The present paper uses a variant of the method of [5], together with results on the cohomology of configuration spaces of $R^{n}$ obtained in [3], to prove the following:

THEOREM 1.4. There does not exist a $k$-regular map of $R^{2}$ into $R^{2 k-\alpha(k)-1}$ where $\alpha(k)$ denotes the number of ones in the dyadic expansion of $k$.

Note that 1.4 gives an improvement over 1.3 in case $n=2$. When $k$ is a power of 2, Example 1.2 shows that 1.4 is best possible.

2. Equivariant maps from configuration spaces to Stiefel manifolds. If $X$ is a topological space, let $F(X, k)$ denote the $k$ th configuration space of $X$, i.e. the subspace of $X^{k}$ consisting of all ordered $k$-tuples of distinct points in $X$. Let $V_{k}\left(R^{N}\right)$ denote the Stiefel manifold of linearly independent (not necessarily orthonormal) $k$-frames in $R^{N}$. The symmetric group $\Sigma_{k}$ acts freely on $F(X, k)$ and $V_{k}\left(R^{N}\right)$ by permuting factors: $\sigma\left(y_{1}, \ldots, y_{k}\right)=\left(y_{\sigma^{-1}(1)}, \ldots, y_{\sigma^{-1}(k)}\right), \sigma \in$ $\Sigma_{k},\left(y_{1}, \ldots, y_{k}\right) \in F(X, k)$ or $V_{k}\left(R^{N}\right)$. A $k$-regular map $f: X \rightarrow R^{N}$ yields a $\Sigma_{k}$-equivariant map $g: F(X, k) \rightarrow V_{k}\left(R^{N}\right)$ given by $g\left(x_{1}, \ldots, x_{k}\right)=$ $\left(f\left(x_{1}\right), \ldots, f\left(x_{k}\right)\right)$.

$\Sigma_{k}$ acts orthogonally on $R^{k}$ by permuting factors. Thus we obtain real $k$-plane bundles

$$
F(X, k) \times_{\Sigma_{k}} R^{k} \rightarrow F(X, k) / \Sigma_{k}
$$

and

$$
V_{k}\left(R^{N}\right) \times_{\Sigma_{k}} R^{k} \rightarrow V_{k}\left(R^{N}\right) / \Sigma_{k}
$$

Proposition 2.1. There exists a $\Sigma_{k}$-equivariant map $F(X, k) \rightarrow V_{k}\left(R^{N}\right)$ if and only if the $k$-plane bundle $F(X, k) \times_{\Sigma_{k}} R^{k} \rightarrow F(X, k) / \Sigma_{k}$ admits an $N$ - k-plane inverse.

Proof. If such an $N-k$-plane inverse existed, there would exist a map $f$ : $F(X, k) \times_{\Sigma_{k}} R^{k} \rightarrow R^{N}$ whose restriction to each fibre is an $R$ monomorphism. Then $g: F(X, k) \rightarrow V_{k}\left(R^{N}\right)$ given by $g(x)=$ $\left(f\left(x, e_{1}\right), \ldots, f\left(x, e_{k}\right)\right)$, where $e_{1}, \ldots, e_{k}$ is the standard basis of $R^{k}$, is $\Sigma_{k}$-equivariant.

The converse follows from the fact that $V_{k}\left(R^{N}\right) \times_{\Sigma_{k}} R^{k} \rightarrow V_{k}\left(R^{N}\right) / \Sigma_{k}$ 
admits an $N-k$-plane inverse. In fact, $f: V_{k}\left(R^{N}\right) \times_{\Sigma_{k}} R^{k} \rightarrow R^{N}$ given by $f\left(y_{1}, \ldots, y_{k} ; t_{1}, \ldots, t_{k}\right)=\sum_{i} t_{i} y_{i}$ is well defined and its restriction to each fibre is an $R$-monomorphism.

COROLlaRY 2.2. If a $k$-regular map $X \rightarrow R^{N}$ exists, then $F(X, k) \times_{\Sigma_{k}} R^{k} \rightarrow$ $F(X, k) / \Sigma_{k}$ admits an $N-k$-plane inverse.

Corollary 2.3. If $X$ admits a Haar system $f_{1}, \ldots, f_{n}$, then the bundle $F(X, n) \times_{\Sigma_{n}} R^{n} \rightarrow F(X, n) / \Sigma_{n}$ is trivial as an $R^{n}$-bundle with group $\Sigma_{n}$.

3. Proof of Theorem 1.4. Write $P_{2, k}$ for the vector bundle $F\left(R^{2}, k\right) \times_{\Sigma_{k}} R^{k}$ $\rightarrow F\left(R^{2}, k\right) / \Sigma_{k}$. By [4, Theorem 1], the Whitney sum of two copies of $P_{2, k}$ is trivial, and so $\bar{w}_{i}\left(P_{2, k}\right)=w_{i}\left(P_{2, k}\right)$ for all $i$. Thus 1.4 will follow from 2.2 and the following:

THEOREM 3.1. $w_{k-\alpha(k)}\left(P_{2, k}\right) \neq 0$.

LeMma 3.2. Let $k$ be a power of 2. Then $w_{k-1}\left(P_{2, k}\right) \neq 0$.

Proof of 3.2. All homology and cohomology groups are with $Z / 2 Z$ coefficients. Let $\rho: B \Sigma_{k} \rightarrow B O(k)$ be induced by the regular representation $\Sigma_{k} \rightarrow O(k)$. The following composite is a classifying map for $P_{2, k}$ :

$$
F\left(R^{2}, k\right) / \Sigma_{k} \stackrel{\sigma_{k}}{\rightarrow} F\left(R^{\infty}, k\right) / \Sigma_{k} \cong B \Sigma_{k} \stackrel{\rho}{\rightarrow} B O(k)
$$

where $R^{\infty}=\operatorname{inj} \lim _{n} R^{n}$ and $\sigma_{k}=\operatorname{inj} \lim _{n} \sigma_{n, k}$ where $\sigma_{n, k}$ is given in [7, p. 35].

Let $C_{n}$ denote the little $n$-cubes operad with $C_{n} X$ the associated construction given by J. P. May [7, p. 13] $\bigodot_{n}(j)$ is equivariantly homotopically equivalent to $F\left(R^{n}, j\right)$ and by $[7$, p. 36], the following diagram commutes:

$$
\begin{array}{lll}
\bigodot_{2}(k) / \Sigma_{k} & \stackrel{\sigma_{k}}{\rightarrow} & \bigodot_{\infty}(k) / \Sigma_{k} \\
\downarrow g_{2} & & \downarrow g \\
F\left(R^{2}, k\right) / \Sigma_{k} & \stackrel{\sigma_{k}}{\rightarrow} & F\left(R^{\infty}, k\right) / \Sigma_{k}
\end{array}
$$

where the top $\sigma_{k}=\operatorname{inj} \lim _{n} \sigma_{n, k}, g_{2}$ is a homotopy equivalence, and $g=$ inj $\lim _{n} g_{n}$ is a homotopy equivalence. Note that $C_{n} S^{0}=\amalg_{j \geqslant 0} \mathcal{C}_{n}(j) / \Sigma_{j}$ and that the map $\sigma_{k}$, on the level of little cubes, is precisely the map $\sigma$ : $C_{2} S^{0} \rightarrow C_{\infty} S^{0}, \sigma=\operatorname{inj} \lim _{n} \sigma_{n}$ (by [7, Theorem 5.2]), restricted to the $k$ th component. We use the observations to compute $\sigma_{k}$ in homology.

Let [1] denote the element in $H_{0}\left(S^{0}\right)$ represented by the non-base-point. By $[3, \S 3], H_{*}\left(C_{n} S^{0}\right)$ is given in terms of Dyer-Lashof operations on [1].

Let $k=2^{i}$ and consider the sequence $I=\left(2^{i-1}, 2^{i-2}, \ldots, 2,1\right)$. By [3, $\S \S 1,4]$, the element $Q^{I}[1]$ is defined in $H_{*}\left(C_{2} S^{0}\right)$ and by the filtration arguments given there, we see that $Q^{I}[1] \in H_{*}\left(\bigodot_{2}(k) / \Sigma_{k}\right)$. Since $\sigma$ is a morphism of $C_{2}$-spaces, we have the formula $\sigma_{k^{*}} Q^{I}[1]=Q^{I}[1]$.

By [8, Theorem 4.7], $\left\langle\rho^{*} w_{k-1}, Q^{I}[1]\right\rangle=1$ where $\langle$,$\rangle denotes the Kronec-$ ker index. Hence 


$$
\begin{aligned}
\left\langle\sigma_{k}^{*} \rho^{*} w_{k-1}, Q^{I}[1]\right\rangle & =\left\langle\rho^{*} w_{k-1}, \sigma_{k^{*}} Q^{I}[1]\right\rangle \\
& =\left\langle\rho^{*} w_{k-1}, Q^{I}[1]\right\rangle=1
\end{aligned}
$$

and so $w_{k-1}\left(P_{2, k}\right)=\sigma_{k}^{*} \rho^{*} w_{k-1} \neq 0$.

Proof of 3.1. Write $k=\sum_{i=1}^{\alpha(k)} j(i)$ where $j(i)=2^{n(i)}, n(1)<n(2)<\cdots<$ $n(\alpha(k))$. We have a map of $k$-plane bundles $f: P_{2, j(1)} \times \cdots \times P_{2, j(\alpha(k))} \rightarrow P_{2, k}$ as follows: Choose pairwise-disjoint open discs $E_{1}, \ldots, E_{\alpha(k)}$ in $R^{2}$. Then we can regard $P_{2, j(i)}$ as

$$
F\left(E_{i}, j(i)\right) \times_{\Sigma_{j(i)}} R^{j(i)} \rightarrow F\left(E_{i}, j(i)\right) / \Sigma_{j(i)} .
$$

Define $f$ by

$$
\begin{array}{r}
f\left(\left(x_{1}, v_{1}\right), \ldots,\left(x_{\alpha(k)}, v_{\alpha(k)}\right)\right)=\left(x_{1}, \ldots, x_{\alpha(k)} ; v_{1}, \ldots, v_{\alpha(k)}\right) \\
\left(x_{i}, v_{i}\right) \in F\left(E_{i}, j(i)\right) \times_{\Sigma_{j(i)}} R^{j(i)} .
\end{array}
$$

Note that for any $j, w_{j}\left(P_{2, j}\right)=0$ since $P_{2, j}$ admits the nowhere-zero section $(x) \mapsto(x ; 1, \ldots, 1)$. Hence

$$
\begin{aligned}
f^{*} w_{k-\alpha(k)}\left(P_{2, k}\right) & =w_{k-\alpha(k)}\left(P_{2, j(1)} \times \cdots \times P_{2, j(\alpha(k))}\right) \\
& =w_{j(1)-1}\left(P_{2, j(1)}\right) \times \cdots \times w_{j(\alpha(k))-1}\left(P_{2, j(\alpha(k))}\right) \neq 0,
\end{aligned}
$$

completing the proof.

\section{REFERENCES}

1. V. G. Boltjanskii, S. S. Ryškov and Ju. A. Šaškin, On k-regular imbeddings and their application to the theory of approximation of functions, Uspehi Mat. Nauk 15 (1960), no. 6 (96), 125-132; English transl., Amer. Math. Soc. Transl. (2) 28 (1963), 211-219.

2. K. Borsuk, On the $k$-independent subsets of the Euclidean space and of the Hilbert space, Bull. Acad. Polon. Sci. Cl. III 5 (1957), 351-356.

3. F. R. Cohen, The homology of $\mathcal{C}_{n+1}$-spaces, $n \geq 0$, Lecture Notes in Math., no. 533, Springer-Verlag, Berlin and New York, 1976, pp. 207-351.

4. F. R. Cohen, M. E. Mahowald and R. J. Milgram, The stable decomposition for the double loop space of a sphere, Proc. of the A. M. S. Summer Institute in Algebraic Topology, Stanford Univ., 1976 (to appear).

5. D. Handel and J. Segal, On k-regular embeddings of spaces in Euclidean space, Fund. Math. (to appear).

6. J. C. Mairhuber, On Haar's theorem concerning Chebychev approximation problems having unique solution, Proc. Amer. Math. Soc. 7 (1956), 609-615.

7. J. P. May, The geometry of iterated loop spaces, Lecture Notes in Math., no. 271, SpringerVerlag, Berlin and New York, 1972.

8. S. Priddy, Dyer-Lashof operations for the classifying spaces of certain matrix groups, Quart. J. Math. Oxford Ser. (2) 26 (1975), 179-193.

9. I. Singer, Best approximation in normed linear spaces by elements of linear subspaces, Springer-Verlag, Berlin and New York, 1970.

School of Mathematics, Institute for Advanced Study, Princeton, New Jersey 08540

Department of Mathematics, Wayne State University, Detroit, Michigan 48202 (Current address of D. Handel)

Current address (F. R. Cohen): Department of Mathematics, Northern Illinois University, DeKalb, Illinois 60115 